\title{
Impact of different grades of anaemia severity during pregnancy on maternal and neonatal outcomes: a prospective study
}

\author{
Sonia Dahiya ${ }^{1}$, Nisha Malik ${ }^{1 *}$, Kiran Pandey ${ }^{2}$, Renu Gupta $^{2}$, \\ Vanamail Perumal ${ }^{3}$, Vikram Singh ${ }^{4}$
}

\begin{abstract}
${ }^{1}$ Department of Obstetrics and Gynaecology, Pt. B.D Sharma PGIMS, Rohtak, Haryana, India ${ }^{2}$ Department of Obstetrics and Gynaecology GSVM Medical College, Kanpur, Uttar Pradesh, India ${ }^{3}$ Department of Statistics \& Demography, All India Institute of Medical Sciences, New Delhi, India ${ }^{4}$ Department of General Surgery, Pt. B.D Sharma PGIMS, Rohtak, Haryana, India
\end{abstract}

Received: 03 April 2019

Accepted: 06 May 2019

\author{
*Correspondence: \\ Dr. Nisha Malik, \\ E-mail: dr.maliknisha@gmail.com
}

Copyright: ( $)$ the author(s), publisher and licensee Medip Academy. This is an open-access article distributed under the terms of the Creative Commons Attribution Non-Commercial License, which permits unrestricted non-commercial use, distribution, and reproduction in any medium, provided the original work is properly cited.

\begin{abstract}
Background: Anaemia in pregnancy is a universal health problem that may cause a number of obstetrical and neonatal complications. This prospective observational study aims to evaluate and compare maternal and neonatal outcomes in different grades of anaemia severity.

Methods: A total of 400 pregnant women with anaemia in third trimester were classified into three groups according to haemoglobin $(\mathrm{Hb})$ levels-group I with $\mathrm{Hb}: 10-10.9 \mathrm{~g} / \mathrm{dl}$, group II with $\mathrm{Hb}: 7-9.9 \mathrm{~g} / \mathrm{dl}$ and group III with $\mathrm{Hb}<7 \mathrm{~g} / \mathrm{dl}$. Maternal and neonatal outcomes of women with different severity of anaemia were analyzed and compared. Two groups means were compared by Student's t-independent test and more than two groups means by one way analysis of variance test followed by post-hoc pairwise comparison using Bonferroni test.

Results: The prevalence of anaemia in the study population was $35.2 \%$. Mild, moderate and severe anaemia were found in 58\% ( $\mathrm{n}=232), 29.0 \%(\mathrm{n}=116)$ and $13 \%(\mathrm{n}=52)$ women respectively. A statistically significant difference in maternal outcomes such as Preterm labor $(\mathrm{p}=0.001)$, Prelabor premature rupture of membranes $(\mathrm{p}=0.044)$, Intrauterine growth restriction $(\mathrm{p}=0.002)$ and postpartum hemorrhage $(\mathrm{p}=0.001)$ was observed amongst the three groups. Cardiac failure occurred in $26.9 \%(n=14)$ and mortality in $13.4 \%(n=7)$ women with severe anaemia. Amongst the neonatal morbidities, the rate of low birth weight, preterm birth, respiratory distress syndrome, septicaemia, pneumonitis and jaundice revealed an increasing trend with rising severity of anaemia which was statistically significant.

Conclusions: Targeted interventions addressing early detection and appropriate treatment in early pregnancy can prevent and avoid dismal maternal and neonatal consequences.
\end{abstract}

Keywords: Anaemia, Pregnancy, Maternal, Neonatal outcome

\section{INTRODUCTION}

Worldwide, anaemia is one of the most prevalent public health problems. It has been estimated by World Health Organisation (WHO) that prevalence of anaemia in pregnancy in developed and developing nations is $14 \%$ and $51 \%$ respectively while in India alone, this number reaches 65 to $75 \% .^{1}$ The data from National Family Health Survey-3 (2005-06) has reported that India falls under the category of "severe public health problem" in WHO classification and has a prevalence rate of anaemia in pregnancy as $58.7 \% .^{2}$ According to WHO, anaemia in pregnancy is defined as haemoglobin $(\mathrm{Hb})$ concentration of less than $11 \mathrm{~g} / \mathrm{dl}$ and a hematocrit of less than $33 \% .^{3}$ 
This medical disorder in pregnancy is not only associated with a number of obstetrical and neonatal complications but also threatens the life of both mother and fetus. It has been linked to $20 \%$ direct maternal deaths and $20-40 \%$ of indirect maternal deaths in India. ${ }^{4,5}$ Anaemia in pregnancy is an important preventable and treatable condition that can bring down the maternal and perinatal mortality rates substantially. This study evaluates the prevalence of anaemia in antenatal women and its impact on maternal and perinatal outcomes according to its severity.

\section{METHODS}

This prospective observational study for one year was conducted in a cohort of women attending the antenatal clinic of the Department of Obstetrics and Gynaecology, GSVM Medical College, Kanpur, Uttar Pradesh, India. All the pregnant women with a singleton pregnancy at a period of gestation $(\mathrm{POG})>28$ weeks with $\mathrm{Hb}$ levels $<11$ $\mathrm{g} / \mathrm{dl}$ at their first visit were recruited for the study. The exclusion criteria included- any history of bleeding disorders, congenital anomalies in fetus, chronic maternal diseases (diabetes mellitus, tuberculosis, heart disease, renal or hepatic disease, chronic hypertension), pregnancy induced hypertension and rhesus factor incompatibility and any episode of vaginal bleeding in the antenatal period. The study was done in accordance with the ethical standards of the institutional committee and with the Helsinki Declaration of 1975, as revised in 2008. A written informed consent was obtained from each participant of the study.

A detailed history was taken that included sociodemographic characteristics, obstetric history, past medical or surgical history, dietary history, antenatal course, any history of iron supplementation or blood transfusion and any relevant family history. Gestational age was calculated from their last menstrual period or earliest ultrasound report whichever applicable. The patient was labelled as booked if she had atleast three antenatal visits or unbooked if otherwise. Thorough general physical examination, systemic examination and antenatal examination was done for each patient. Baseline investigations were done in all patients including complete hemogram, peripheral blood smear, liver function tests, renal function tests, viral markers, urine microscopy, blood sugar, antenatal ultrasound if indicated. Serum iron studies and $\mathrm{Hb}$ electrophoresis could not be done due to their non availability in the institute.

Hemoglobin estimation was done first at the time of enrolment followed by repeat testing 3 weeks after starting iron therapy (to see response), then monthly till delivery and on third postpartum day. Women were classified into three groups according to severity of anaemia at the first visit - group I with mild anaemia ( $\mathrm{Hb}$ : 10-10.9 g/dl), group II with moderate anaemia (Hb: 7-9.9 $\mathrm{g} / \mathrm{dl})$ and group III with severe anaemia ( $\mathrm{Hb}<7 \mathrm{~g} / \mathrm{dl})$.
Appropriate treatment for anaemia was started according to their baseline $\mathrm{Hb}$ values and POG. Any maternal complications arising due to anaemia in antenatal period, during labour and postpartum period upto day 7 were noted. Baby details at the time of birth and early neonatal problems were also recorded. These factors were then compared among the three groups to see the effect according to the severity of anaemia. In the study, baby born with weight $<2500 \mathrm{~g}$ was considered as low birth weight (LBW) and $<37$ weeks of gestation was considered as preterm birth (PTB).

Data analysis was carried out using SPSS IBM version 20.0 (Armonk, NY: IBM Corp). Continuous variables were tested for normality assumption using KolmogorovSmirnov test. Descriptive statistics such as mean, standard deviation (SD) and range values were calculated. Comparison of two groups means were tested by Student's t-independent test and more than two groups means were compared by one way analysis of variance test followed by post-hoc pairwise comparison using Bonferroni test. Qualitative data were expressed as frequency and percent values. Frequency data across categories were compared using Chi-square or Fishers's Exact test as appropriate. For all statistical tests a two sided probability of $\mathrm{p}<0.05$ was considered as statistical significance.

\section{RESULTS}

A total of 1180 pregnant women were screened at antenatal clinic, of whom 416 women were diagnosed with anaemia, making the prevalence of this condition in study population as $35.2 \%$. All were categorised into three groups according to the severity of anaemia as described earlier. Figure 1 explains the consort flow diagram of the study design. The outcomes were studied in total 400 pregnant women; 58\% $(n=232), 29.0 \%$ $(\mathrm{n}=116)$ and $13 \%(\mathrm{n}=52)$ in group I, group II and III respectively. Table 1 indicates the socio-demographic and obstetrical characteristics of women in three groups. The mean \pm SD (range) age of women was 25.0 33.3 (19$35)$ years. Though most of women belonged to age group between 21-25 years, there were $3.5 \% \quad(n=14)$ women who were 18-20 years of age. It was observed that there was a significantly higher proportion of multiparous women with rural background and low socio economic class in group III as compared to the other two groups $(\mathrm{p}=0.001)$. Ninety-six percent $(\mathrm{n}=50)$ women with severe anaemia were unbooked as compared to $41.3 \%$ and $67.2 \%$ in mild and moderate group respectively $(\mathrm{p}=0.001)$. The type of anaemia observed on peripheral blood film was microcytic hypochromic in $59.5 \%$ $(n=238)$, normocytic normochromic in $14.5 \%(n=58)$ and dimorphic in $26 \%(\mathrm{n}=104)$ women. It was also an important observation that $36.5 \% \quad(n=19)$ women with severe anaemia delivered before 37 weeks POG which was significantly higher as compared to other two groups $(\mathrm{p}=0.007)$. 
Table 1: Socio-demographic and obstetrical characteristics of women in different groups $(\mathrm{n}=400)$.

\begin{tabular}{|c|c|c|c|c|c|}
\hline \multicolumn{2}{|l|}{ Characteristic } & $\begin{array}{l}\text { Group I } \\
232(58 \%)\end{array}$ & $\begin{array}{l}\text { Group II } \\
116(29 \%)\end{array}$ & $\begin{array}{l}\text { Group III } \\
52(13 \%)\end{array}$ & $P$ value \\
\hline & & n $(\%)$ & n $(\%)$ & n $(\%)$ & \\
\hline \multicolumn{2}{|l|}{ Mean $\mathrm{Hb}(\mathrm{g} / \mathrm{dl})$} & $\begin{array}{l}10.3 \pm 0.2 \\
(10-10.9)\end{array}$ & $\begin{array}{l}7.8 \pm 0.8 \\
(7-9.8)\end{array}$ & $\begin{array}{l}5.09 \pm 1.2 \\
(2-6.8)\end{array}$ & 0.001 \\
\hline \multirow{4}{*}{ Age (years) } & $18-20$ & $10(4.3)$ & $3(2.6)$ & $1(1.9)$ & \multirow{4}{*}{0.003} \\
\hline & $21-25$ & $154(66.8)$ & $98(84.5)$ & $38(73.1)$ & \\
\hline & $26-30$ & $34(14.6)$ & $4(3.4)$ & $2(3.8)$ & \\
\hline & $>30$ & $34(14.6)$ & $11(9.5)$ & $11(21.1)$ & \\
\hline \multirow{2}{*}{ Parity } & Primipara & $100(43.1)$ & $42(36.2)$ & $2(3.8)$ & \multirow{2}{*}{0.001} \\
\hline & Multipara & $132(56.9)$ & $74(63.8)$ & $50(96.1)$ & \\
\hline \multirow{3}{*}{$\begin{array}{l}\text { POG at delivery } \\
\text { (weeks) }\end{array}$} & $28-30.6$ & $14(6.0)$ & $9(7.7)$ & $9(17.3)$ & \multirow{3}{*}{0.007} \\
\hline & $31-36.6$ & $20(8.6)$ & $15(12.9)$ & $10(19.2)$ & \\
\hline & $>=37$ & $198(85.3)$ & $92(79.3)$ & $33(63.4)$ & \\
\hline \multirow{2}{*}{ Residence } & Rural & $152(65.5)$ & $90(77.6)$ & $42(80.7)$ & \multirow{2}{*}{0.016} \\
\hline & Urban & $80(34.5)$ & $26(22.4)$ & $10(19.2)$ & \\
\hline \multirow{3}{*}{ Socio economic status } & Lower & $14(6.0)$ & $32(27.6)$ & 35 (67.3) & \multirow{3}{*}{0.001} \\
\hline & Middle & $132(56.9)$ & $68(58.6)$ & $15(28.8)$ & \\
\hline & Upper & $86(37.0)$ & $16(13.8)$ & $2(3.8)$ & \\
\hline \multirow{2}{*}{ Booking status } & Booked & $136(58.6)$ & $38(32.7)$ & $2(3.8)$ & \multirow{2}{*}{0.001} \\
\hline & Unbooked & $96(41.3)$ & $78(67.2)$ & $50(96.1)$ & \\
\hline \multirow{3}{*}{ Mode of delivery } & SVD & $198(85.3)$ & $94(81.0)$ & $42(80.7)$ & \multirow{3}{*}{0.732} \\
\hline & $\begin{array}{l}\text { Instumental } \\
\text { delivery }\end{array}$ & $10(4.3)$ & $6(5.1)$ & $2(3.8)$ & \\
\hline & Caesarean delivery & $24(10.3)$ & $16(13.8)$ & $8(15.4)$ & \\
\hline
\end{tabular}

*SVD-spontaneous vaginal delivery

Table 2: Comparison of maternal complications in different groups.

\begin{tabular}{|lllll|}
\hline Complication & Group I & Group II & Group III & P value \\
\hline & $\mathbf{2 3 2}(\mathbf{5 8} \%)$ & $\mathbf{1 1 6}(\mathbf{2 9} \%)$ & $\mathbf{5 2}(\mathbf{1 3} \%)$ & \\
\hline Preterm labor & $39(16.8)$ & $\mathbf{n}(\mathbf{\%})$ & $\mathbf{n}(\mathbf{\%})$ & 0.001 \\
\hline PPROM & $18(7.7)$ & $13(23.2)$ & $21(40.4)$ & 0.044 \\
\hline Chorioamnionitis & $8(3.4)$ & $8(6.8)$ & $10(19.2)$ & 0.106 \\
\hline PIH & $19(8.1)$ & $8(6.9)$ & $4(7.6)$ & 0.963 \\
\hline IUGR & $40(15.9)$ & $35(30.2)$ & $16(30.8)$ & 0.002 \\
\hline PPH & $8(3.4)$ & $16(13.8)$ & $14(26.9)$ & 0.001 \\
\hline Retained placenta & 0 & $1(0.8)$ & $2(3.8)$ & 0.017 \\
\hline Vulvovaginal hematoma & 0 & $1(0.8)$ & $2(3.8)$ & 0.017 \\
\hline Puerperal pyrexia & $6(2.6)$ & $15(12.9)$ & $8(15.3)$ & 0.001 \\
\hline Wound infection & $21(9.0)$ & $20(17.2)$ & $14(26.9)$ & 0.001 \\
\hline Cardiac failure & 0 & 0 & $14(26.9)$ & 0.001 \\
\hline Mortality & 0 & 0 & $7(13.4)$ & 0.001 \\
\hline
\end{tabular}

* PPROM- Prelabor premature rupture of membranes $\uparrow$ PIH-pregnancy induced hypertension $\$$ IUGR-intrauterine growth restriction $\S$ PPH-postpartum hemorrhage

Table 2 and 3 compares the maternal and neonatal complications arising in women in different grades of anaemia. It was found that there was statistically significant difference in maternal outcomes such as preterm labor $(\mathrm{PTL}) ; \mathrm{p}=0.001$, prelabor premature rupture of membranes (PPROM); $\mathrm{p}=0.044$, Intrauterine growth restriction (IUGR); $\mathrm{p}=0.002$ and postpartum hemorrhage $(\mathrm{PPH}) ; \mathrm{p}=0.001$ amongst the three groups. As anaemia got worsened in severity, the magnitude of maternal complications also increased. The rate of puerperal pyrexia and wound infection were also significantly more in group III ( $\mathrm{p}=0.001)$. Cardiac failure occurred in $26.9 \%$ $(n=14)$ women with severe anaemia whereas no such case was found in the other two groups. Seven women $(13.4 \%)$ with severe anaemia succumbed to death despite aggressive resuscitation efforts. 
Table 3: Comparison of neonatal morbidities in different groups.

\begin{tabular}{|lllll|} 
& Group I & Group II & Group III & P value \\
\hline & $\mathbf{2 3 2}(\mathbf{5 8} \%)$ & $\mathbf{1 1 6 ( 2 9 \% )}$ & $\mathbf{5 2 ( 1 3 \% )}$ & $\mathbf{n}(\mathbf{\%})$ \\
\hline LBW & $\mathbf{n}(\mathbf{\%})$ & $\mathbf{n}(\mathbf{\%})$ & $\mathbf{2 8}(53.8)$ & 0.001 \\
\hline Preterm birth & $74(31.9)$ & $62(53.4)$ & $19(36.5)$ & 0.001 \\
\hline RDS & $34(14.7)$ & $24(20.7)$ & $14(26.9)$ & 0.025 \\
\hline Birth asphyxia & $28(12.0)$ & $18(15.5)$ & $10(19.3)$ & 0.120 \\
\hline Septicaemia & $25(10.7)$ & $20(17.2)$ & $16(30.7)$ & 0.001 \\
\hline Pneumonitis & $10(4.3)$ & $13(11.2)$ & $8(15.3)$ & 0.013 \\
\hline Jaundice & $10(4.3)$ & $10(8.6)$ & $12(23.0)$ & 0.012 \\
\hline
\end{tabular}

* LBW-low birth weight $\uparrow$ RDS-respiratory distress syndrome.

Table 4: Comparison of perinatal deaths in three groups.

\begin{tabular}{|lllll|}
\hline Perinatal deaths & Group I & Group II & Group III & P value \\
\hline & $\mathbf{2 3 2}(\mathbf{5 8 \%})$ & $\mathbf{1 1 6 ( 2 9 \% )}$ & $\mathbf{5 2 ( 1 3 \% )}$ & $\mathbf{n}(\mathbf{\%})$ \\
\hline IUD & $\mathbf{n}(\mathbf{\%})$ & $\mathbf{n}(\mathbf{\%})$ & $\mathbf{5 ( 9 . 6 )}$ & 0.002 \\
\hline FSB & $2(0.8)$ & $1(0.8)$ & $6(11.5)$ & 0.022 \\
\hline END & $6(2.6)$ & $4(3.4)$ & $4(7.6)$ & 0.014 \\
\hline
\end{tabular}

* IUD-intrauterine death $\uparrow$ FSB-fresh still birth $\$$ END-early neonatal death

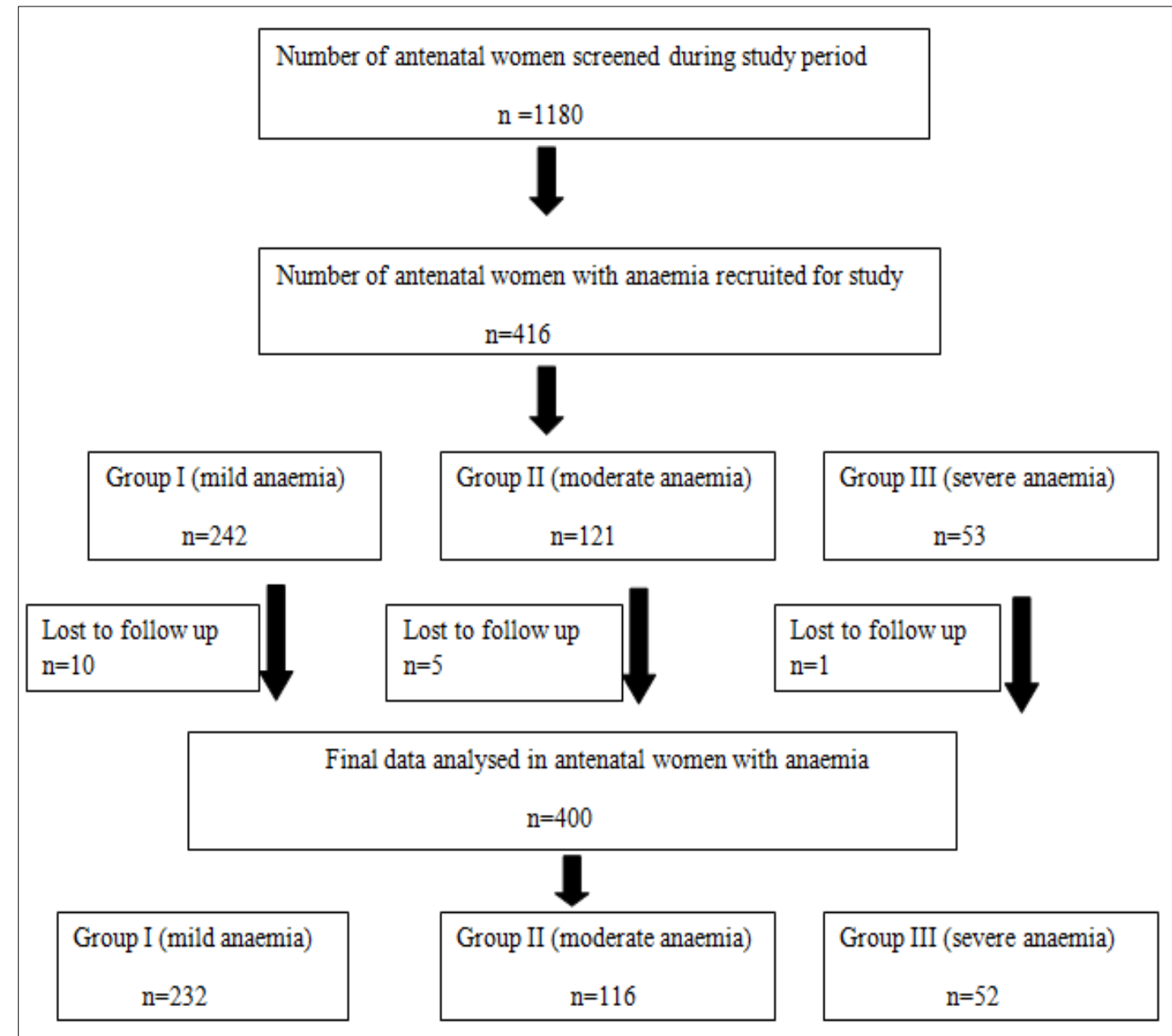

Figure 1: Consort flow diagram of study design. 
Amongst the neonatal morbidities, the rate of LBW, PTB, Respiratory distress syndrome, septicaemia, pneumonitis and jaundice revealed an increasing trend with rising severity of maternal anaemia which was statistically significant. When the perinatal deaths were compared in three groups as shown in Table 4, it was found that there were significantly higher number of intrauterine deaths (IUD); $\mathrm{p}=0.002$, fresh still births; $\mathrm{p}=0.022$ and early neonatal deaths; $\mathrm{p}=0.014$ ) in group III. Out of the five IUDs in severely anaemic women, three had severe IUGR, one had meconium stained liquor and one had abruptio placentae.

\section{DISCUSSION}

This study has evaluated the effect of one of the most common medical disorder of pregnancy that may prove to be life threatening for the mother and fetus. The estimated prevalence rate of anaemia in pregnancy in current study was $35.2 \%$. The overall pooled prevalence of anaemia in low and middle income countries as observed by Rahman et al (2016) in a systematic review and metanalysis was $42.7 \%$ (95\% CI: $37.0 \%, 48.4 \%)$ while in India alone, it was $59 \% .^{6}$ The prevalence of mild and moderate-to-severe anaemia in India as reported in a study by Perumal was $24.1 \%$ (95\% CI: $23.0-25.3 \%)$ and $30.5 \%$ (95\% CI: $29.3-31.8 \%$ ), respectively. ${ }^{7}$

The population representative data between 1995 and 2011 has shown highest prevalence of anaemia in pregnant and non pregnant women from South Asia (47\% of reproductive-age women and $52 \%$ of pregnant women) and Central and West Africa whereas it was $16 \%$ of women and $22 \%$ of pregnant women in high income countries. $^{6,8}$ Various socio-demographic, clinical and cultural factors play role in influencing the maternal health in developing nations like India such as poverty, lack of knowledge, younger age at marriage, high parity, infections, poor access to health services. ${ }^{9}$ The study has reflected that anaemia was more severe in unbooked mutiparous women with lower socioeconomic class and rural background. Perumal has also reported $30 \%$ excess risk of moderate-to-severe anaemia in rural areas $(32.4 \%)$ than urban areas $(27.3 \%){ }^{7}$

Maternal anaemia has been linked to a number of medical and obstetrical complications in pregnancy and the outcomes worsen with an increase in severity of anaemia. The etiology of association of PTL with anaemia is still unclear. Studies have suggested that role of maternal infection, chronic hypoxia and oxidative stress associated with iron deficiency that can activate the maternal or fetal hypothalamic-pituitary-adrenal axis and eventually causing preterm parturition. ${ }^{10,11}$ Infection may be involved in causing PPROM which in turn can also lead to preterm labour. Various biological mechanisms have been proposed linking anaemia with IUGR. The environment of chronic hypoxia and oxidative stress as a result of low $\mathrm{Hb}$ concentration can cause fetal growth restriction. Another theory says that iron deficiency results in increased norepinephrine secretion which in turn stimulates production of corticotropin-releasing hormone that can restrict fetal growth. ${ }^{10,12}$ This association with preterm delivery and IUGR in turn explains the mechanism of LBW newborns in maternal anaemia and its related neonatal morbidities.

The rate of PTL in the current study was $40.4 \%$ in severely anaemic women (which transformed into a PTB rate of $36.5 \%$ ) whereas $16.8 \%$ and $23.2 \%$ in mild and moderate anaemia. The rate of IUGR and LBW in severely anaemic women was $30.8 \%$ and $53.8 \%$ respectively. Results from a number of studies are consistent with the findings of association of preterm delivery and subsequent LBW with maternal anaemia. The systematic review and metanalysis by Rahman et al has reported that anaemia in pregnant women attributed to a significantly higher risks of LBW (RR: $1.31 ; 95 \%$ CI: 1.13 , 1.51), PTB (RR: 1.63; 95\% CI: 1.33, 2.01), perinatal mortality (RR: $1.51 ; 95 \% \mathrm{CI}: 1.30,1.76)$, and neonatal mortality (RR: 2.72; 95\% CI: 1.19, 6.25) in low and middle income countries. ${ }^{6}$ The same study has reported the data specifically in India as- LBW (RR: 1.13; $95 \%$ CI: 0.92, 1.38), PTB (RR: 1.63 ; 95\% CI: (0.88, 3.04)), perinatal mortality (RR: 1.70 ; $95 \%$ CI: 0.66,4.38). ${ }^{6}$ Although an earlier reported literature by Rohilla et al has shown a high incidence $(17.7 \%$ ) of pregnancy induced hypertension in severe anemia, it was only $7.7 \%$ in the present study and was not significantly different among different groups. ${ }^{13}$

Anaemia is not only responsible for antenatal complications but also linked with an increased risk of $\mathrm{PPH}$ and other postpartum problems. It has been hypothesized that anaemia may cause reduced uterine blood flow and uterine muscle strength that can lead to uterine dysfunction or inertia resulting in PPH. ${ }^{14}$ Also, the severely anaemic women fail to tolerate any excessive blood loss during delivery and have high chances of landing up into cardiac failure. Our data was consistent with these findings as PPH was more often seen in women with moderate and severe anaemia than those with mild deficiency. The incidence of puerperal pyrexia and wound infection (caesarean scar or episiotomy site) was also significantly greater in severely anaemic women. High rate of PPH in the current study (26.9\%) in women with severe anaemia in was comparable to that observed by Rohilla et al which was $25 \% .^{13}$ Severe anaemia contributed to $13.4 \%$ maternal deaths in present study which was significantly more than that observed in previous study by Rohilla et al which was $6.25 \% .{ }^{13}$ Such poor outcome even in a tertiary care hospital may be explained by factors such as lack of awareness and education, delay in seeking medical care, critical condition of patient at time of admission, delayed supply of appropriate blood and blood products.

To conclude, anaemia in pregnancy is a serious global health problem. The study revealed that this condition may cause disastrous consequences for the mother and 
fetus. Targeted interventions addressing early detection and appropriate treatment in early pregnancy can prevent and avoid dismal maternal and neonatal outcomes. Implementing measures for prevention and control of anaemia in adolescence should be a priority.

\section{ACKNOWLEDGMENTS}

We acknowledge the colleagues who supported us while conducting the study. We also acknowledge the patients who participated in the study.

Funding: No funding sources Conflict of interest: None declared

Ethical approval: The study was approved by the Institutional Ethics Committee

\section{REFERENCES}

1. Kalaivani K. Prevalence and consequences of anaemia in pregnancy. Indian $\mathbf{J}$ Med Res. 2009; 130:627-33.

2. International Institute of Population Sciences and ORC Macro. National Family Health Survey - 3. International Institute of Population Sciences, Mumbai. Available at: http://www.iipsindia.org/ nfhs3.html. Accessed on 3 January 2019.

3. WHO, Iron deficiency anaemia: assessment, prevention and control. WHO/NHD/ 01.3, Geneva, 2001.

4. Malagi U, Reddy M, Naik R. Evaluation of national nutritional control programme in Dharward (Karnataka). J Hum Ecol. 2006;20(4):279-81.

5. Kumar A, Jain S, Singh NP, Singh T. Oral versus high dose parenteral iron supplementation in pregnancy. Int J Gynecol Obstet. 2005;89:7-13.

6. Rahman MM, Abe SK, Rahman MS, Kanda M, Narita S, Bilano V, et al. Maternal anemia and risk of adverse birth and health outcomes in low- and middle-income countries: systematic review and meta-analysis. Am J Clin Nutr. 2016;103(2):495504.

7. Perumal V. Reproductive risk factors assessment for anaemia among pregnant women in India using a multinomial logistic regression model. Trop Med Int Health. 2014;19(7):841-51.

8. Stevens GA, Finucane MM, De-Regil LM, Paciorek CJ, Flaxman SR, Branca F, Peña-Rosas JP, Bhutta ZA, Ezzati M. Global, regional, and national trends in haemoglobin concentration and prevalence of total and severe anaemia in children and pregnant and non-pregnant women for 1995-2011: a systematic analysis of population-representative data. Lancet Glob Health. 2013;1:e16-25.

9. Rao S, Joshi S, Bhide P, Puranik B, Kanade A. Social dimensions related to anaemia among women of childbearing age from rural India. Public Health Nutr. 2011;14(2):365-72.

10. Allen LH. Biological mechanisms that might underlie iron's effects on fetal growth and preterm birth. J Nutr 2001;131:581s-89s.

11. Zhang Q, Ananth CV, Li Z, Smulian JC. Maternal anaemia and preterm birth: a prospective cohort study. Int J Epidemiol. 2009;38(5):1380-9.

12. Kozuki N, Lee AC, Katz J Moderate to Severe, but Not Mild, Maternal Anemia, Is Associated with Increased Risk of Small-for-Gestational-Age Outcomes. J Nutr. 2012;142(2):358-62.

13. Rohilla M, Raveendran A, Dhaliwal LK, Chopra S. Severe anaemia in pregnancy: A tertiary hospital experience from northern India. J Obstet Gynaecol. 2010;30(7):694-6.

14. Kavle JA, Stoltzfus RJ, Witter F, Tielsch JM, Khalfan SS, Caulfield LE. Association between Anaemia during Pregnancy and Blood Loss at and after Delivery among Women with Vaginal Births in Pemba Island, Zanzibar, Tanzania. J Health Popul Nutr. 2008;26(2):232-40.

Cite this article as: Dahiya S, Malik N, Pandey K, Gupta R, Perumal V, Singh V. Impact of different grades of anaemia severity during pregnancy on maternal and neonatal outcomes: a prospective study. Int J Reprod Contracept Obstet Gynecol 2019;8:221520 . 